Check for updates

Cite this: RSC Adv., 2017, 7, 26722

\title{
Improved oxygen reduction activity of carbon nanotubes and graphene through adenine functionalization
}

\author{
Youze Xu, ${ }^{a}$ Caili Chen, ${ }^{a}$ Mo Zhou, ${ }^{b}$ Guangyi FU, ${ }^{a}$ Yuanyuan Zhao ${ }^{a}$ \\ and Yuehui Chen (D)*b
}

\begin{abstract}
Here we demonstrated a simple and straightforward way which can significantly improve the ORR activity of single walled carbon nanotubes (SWNTs) and graphene through adenine functionalization. Adenine can interact with CNTs and graphene's carbon planes, which draw electrons from adenine to make the N species form pyrrolic and graphitic- $\mathrm{N}$, similar to $\mathrm{N}$ species of $\mathrm{N}$ doped materials. The activity for the ORR at a potential of $-0.25 \mathrm{~V}$ vs. SCE was improved by 4.5 and 1.8 times, and the half-wave potential was around $-0.25 \mathrm{~V}$ and $0.22 \mathrm{~V}$ vs. SCE for adenine functionalized SWNTs and graphene, which is 120 and $90 \mathrm{mV}$ lower than Pt-C, respectively. Even though the activity and onset potential is smaller than that of commercial $\mathrm{Pt}-\mathrm{C}$, the results provide a simple and straightforward way to improve the activity of carbon materials through molecular functionalization in exploring C-based metal free electrocatalysts for ORR with better fuel selectivity, better $\mathrm{CO}$ tolerance and stability.
\end{abstract}

Received 9th March 2017

Accepted 11th May 2017

DOI: $10.1039 / c 7 r a 02865 b$

rsc.li/rsc-advances

doping normally involve high temperature or hash chemical

\section{Introduction}

Fuel cells powered by hydrogen are an ideal solution for nonpolluting vehicles and portable applications. ${ }^{1-3}$ Extensive research and development in the past decades have delivered fuel cells with impressive performance. ${ }^{1,2}$ However, the cathode ORR is still six or more orders of magnitude slower than the anode hydrogen oxidation reaction, which thus limits performance, ${ }^{1,2}$ resulting in almost all research and development focusing on improving the cathode catalysts. Most ORR catalysts used today are based on Pt or Pd in the form of nanoparticles (NPs) dispersed on carbon supports. ${ }^{2,4-7}$ However the high price and the limited availability of these scarce noble metals are the major challenges that we face in paving the way to bring fuel cell to commercial viability, ${ }^{2,6}$ even though novel structures, such as core-shell structures with monolayer or multi-layer noble metals, ${ }^{8-11}$ Pt-skin structure and extended surface area $^{12}$ etc. were developed with the aim of a better utilization of the noble metals.

Carbon material based metal free catalysts have attracted great attention recently for their low cost, better selectivity and long-term stability. ${ }^{13}$ The active sites for ORR are mainly revealed through doping carbon materials with heteroatoms such as $\mathrm{N},{ }^{14,15}$ B. ${ }^{16}$ Nitrogen doped carbon materials are proved highly effective electrocatalysts for ORR..$^{13,15,17}$ While, nitrogen

${ }^{a}$ Hunan Research Academy of Environment Sciences, Changsha 410004, China. E-mail: youzexing@163.com; 1134081231@qq.com

${ }^{b}$ Department of Environmental Science and Engineering, Xiangtan University, Xiangtan City 411105, China. E-mail: 61080510@qq.com processes. Kannan et al. studied the ORR activity of functionalized carbon nanotubes with different functional groups, such as sulfonic acid and phosphonic acid. Functionalized CNTs show significantly enhanced activity towards the ORR, while CNTs without such surface functional groups do not reveal any such special ORR activity. ${ }^{18}$ Hexadecyl trimethyl ammonium bromide (CTAB)-functionalized graphene oxide (GO) and multiwalled carbon nanotubes (MWCNT) in CTAB and chitosan matrix (CTAB/GO/MCWNT/CS) exhibits a significant synergistic catalytic effect on oxygen reduction reaction. ${ }^{19}$ Wang et al. demonstrated that polyelectrolyte functionalized CNT and graphene could act as metal free electrocatalysts for ORR through the intermolecular charge-transfer from the CNT to the functionalized PDDA. ${ }^{20,21}$ Poly (diallydimethylammonium chloride) (PDDA), having a strong electron-withdrawing ability to withdraw electrons from CNT and graphene, facilitate the ORR catalytic activity of nitrogen free CNT adsorbed with the PDDA chain. ${ }^{21}$ This work clearly indicates that the important role of intermolecular charge transfer to ORR of nitrogen-free CNTs can be applied to other carbon materials for the development of various other metal-free efficient ORR catalysts. ${ }^{22,23}$ Compared with N doped CNT and graphene, polyelectrolyte functionalized CNT provide a simple and easy approach to develop cheap and metal free ORR catalysts.

Here, we discovered that adenine, a nucleobase (a purine derivative) without ORR activity, functionalized SWNT and graphene can significantly improve the activity for ORR compared with bare SWNT and graphene. The improved ORR activity is attributed to the formation of pyrrolic and graphitic $\mathrm{N}$ 
due to charge transfer from $\mathrm{N}$ species of adenine to SWNT or graphene.

\section{Experimental}

\section{Preparation of adenine functionalized carbon materials}

Materials used in this experiment include SWNT (Nanostructured \& Amorphous Materials, Inc. USA), graphene (Beijing Dk Nano technology Co., LTD), nafion solution (5\% in isopropanol and water), ethanol (Sigma-Aldrich), and adenine (structure show in Fig. 1 inserted, molecular weight 135, SigmaAldrich). The as-received SWNT and graphene were purified following the procedure reported: ${ }^{24} 200 \mathrm{mg}$ graphene or SWNT was dispersed in $50 \mathrm{~mL} \mathrm{HCl}$ acid (30 wt\%) before being ultrasonicated for $1 \mathrm{~h}$. The dispersion was separated and the sludge was dispersed in another $50 \mathrm{~mL} \mathrm{HCl}$ acid ( $30 \mathrm{wt} \%$ ), followed by stirring overnight. Then the dispersion was separated using a centrifuge before being washed by DI water for 3 times. The purified graphene or SWNT was dried and collected for further use. All other chemicals are used without further purification unless specified.

Adenine functionalized SWNT and graphene were prepared as follow: $30 \mathrm{mg}$ purified SWNT or graphene then were ultrasonicated in $100 \mathrm{~mL}$ ultrapure water for $1 \mathrm{~h}$ in presence of $0.2 \mathrm{mg} \mathrm{mL}{ }^{-1}$ adenine and followed by stirring for $12 \mathrm{~h}$. The functionalized SWNT or graphene were filtered and washed with ultrapure water for several times to remove the excess adenine (Fig. 1 shows the schematic of material preparation by taking SWNTs as an example). The adenine functionalized graphene were prepared in the same way. The products were denoted as A/SWNT and A/graphene respectively.

\section{Characterization}

Thermo gravimetric (TG, Q5000) analyses were performed under air upon equilibration at $100{ }^{\circ} \mathrm{C}$ for $15 \mathrm{~min}$, followed by a ramp of $10{ }^{\circ} \mathrm{C} \min ^{-1}$ up to $700{ }^{\circ} \mathrm{C}$. The Raman spectra were recorded in air at room temperature using a Perkin-Elmer GX Raman spectrometer with a back-scattered configuration and equipped with a Nd:YAG laser at $1064 \mathrm{~nm}$ as its light source for Raman. Pristine SWNT and graphene were characterized using a scanning electron microscope (SEM, Zesis Neon $40 \mathrm{EsB}$ ) and a transmission electron microscope (TEM, JEOL JEM-2000EX) with operating at $200 \mathrm{kV}$. The X-ray photoelectron spectroscopy (XPS) measurements were carried out on a XPS apparatus (Kratos AXIS Ultra DLD) with pass energy of $40 \mathrm{eV}$.

The electrochemical measurements were conducted in a standard electrochemical cell using a Princeton potentiostat

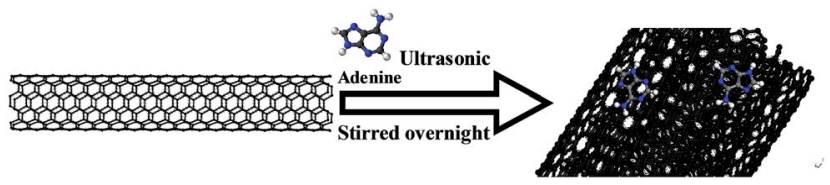

Fig. 1 Schematic of preparation of adenine functionalized carbon nanotubes.
Versastat 3, USA. Pt foil $\left(3.0 \mathrm{~cm}^{2}\right)$ and saturated calomel electrode (SCE) were used as the counter and reference electrodes, respectively. All potentials in the present study were given versus SCE electrode. The cyclic voltammograms (CVs) were measured at a scan rate of $50 \mathrm{mV} \mathrm{s}^{-1}$ in the $0.1 \mathrm{M} \mathrm{KOH}$ solution before saturated with $\mathrm{O}_{2}$ or $\mathrm{N}_{2}$ for $15 \mathrm{~min}$. The linear sweep voltammetry (LSV) were tested at $10 \mathrm{mV} \mathrm{s}^{-1}$ in oxygen saturated $0.1 \mathrm{M}$ $\mathrm{KOH}$ solution. The kinetic analyses of ORR performance are carried out by the Koutecky-Levich equation. ${ }^{21}$

\section{Results and discussion}

The purified SWNT and graphene were firstly characterized using SEM and TEM shown in Fig. 2. The results show the SWNT form bundles (Fig. 2A and B), and the graphene show a typical two-dimensional property with size up to several micrometers (Fig. 2C and D). No metal particles were observed, indicating the high purity of the SWNT and graphene. The adenine functionalized SWNT and graphene were characterized by thermo-gravimetric and Raman analysis. Fig. 3 shows the thermo-gravimetric analysis (TGA) curves recorded under argon for adenine, SWNT, graphene, A/SWNT and A/graphene. The adenine starts to decompose at $260^{\circ} \mathrm{C}$ and totally decomposed at $350{ }^{\circ} \mathrm{C}$. SWNT and graphene are thermal stable, which are start to lose weight at $350{ }^{\circ} \mathrm{C}$. Consequently, the mass loss around $300-350{ }^{\circ} \mathrm{C}$ in the case of A/SWNT and A/graphene can be attributed to the decomposition of adenine indicating the amount of adenine that functionalized on SWNT and graphene. The result shows adenine that supported on SWNT and graphene accounts for about $10 \%$ and $4.1 \%$ respectively. Raman spectrum in Fig. 4 provided further information for adenine functionalized SWNT and graphene. The band near $1300 \mathrm{~cm}^{-1}$ has been called the D-band ${ }^{25,26}$ which is activated by disorder in the $\mathrm{sp}^{2}$ carbon network. The band at $1590 \mathrm{~cm}^{-1}$ is called the Gband ${ }^{25}$ which stems from the symmetry of the SWNT and graphene. The $I_{\mathrm{D}} / I_{\mathrm{G}}$ is 0.16 and 0.14 for SWNT and A/SWNT, and

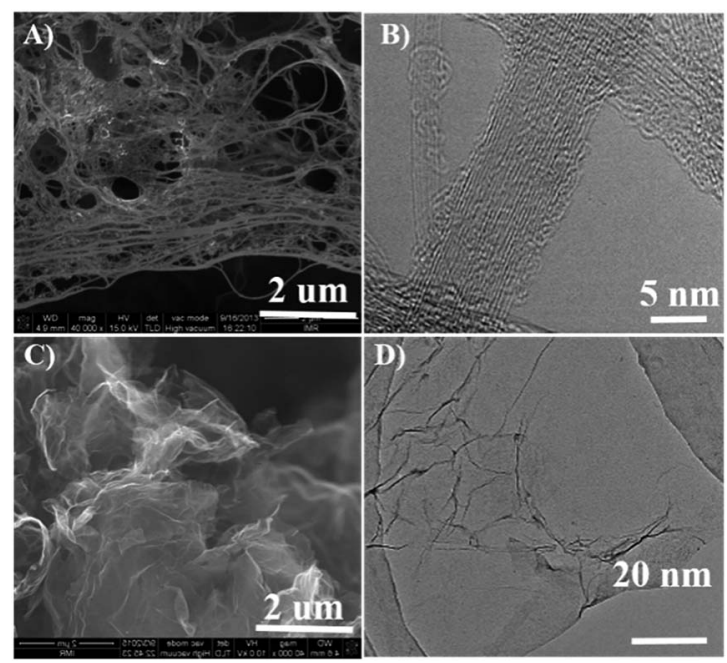

Fig. 2 The (A) SEM and (B) TEM images of SWCNT, and the (C) SEM and (D) TEM of graphene. 

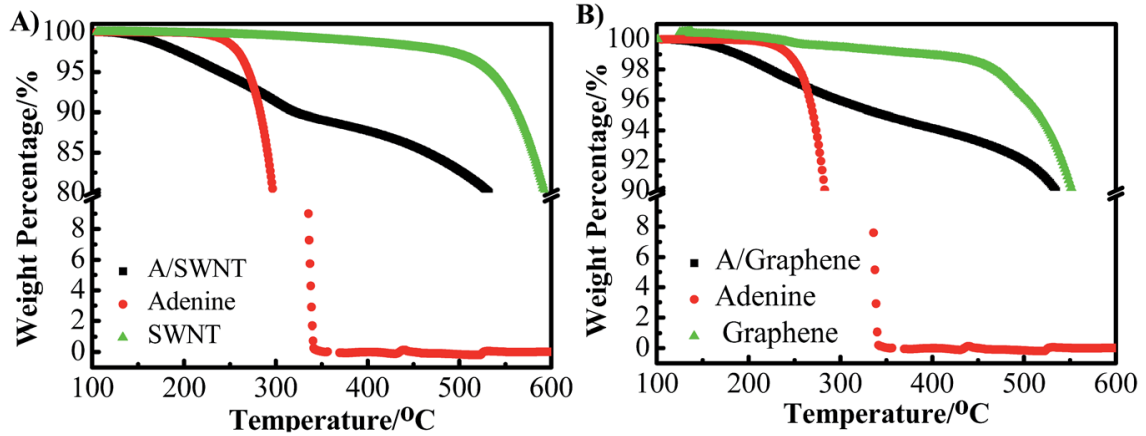

Fig. 3 TGA measurements of (A) adenine, SWNT, A/SWNT and (B) adenine, graphene and A/graphene.
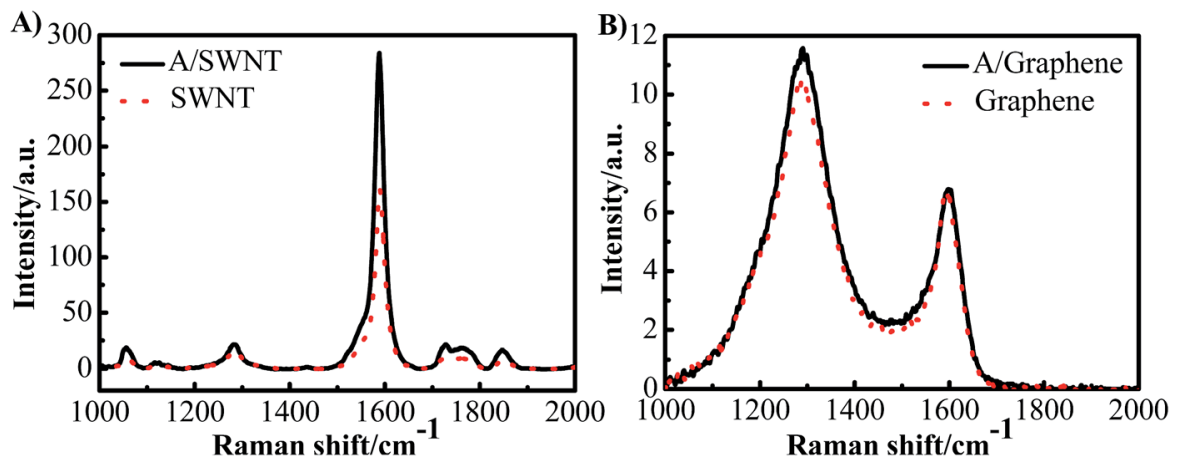

Fig. 4 Raman spectra of (A) SWNT, A/SWNT and (B) graphene and A/graphene.

2.63 and 2.27 for graphene and $\mathrm{A} /$ graphene respectively. The decrease of corresponding $I_{\mathrm{D}} / I_{\mathrm{G}}$ intensity of adenine functionalized SWNT and graphene indicates that adenine functionalization through $\pi-\pi$ interaction can increase of symmetry of SWNT and graphene.

The nature of A/SWNT and A/graphene was further identified by X-ray photoelectron spectroscopy (XPS) with peak deconvolution (Fig. 5). XPS results show that two peaks with binding energy around $284.5 \mathrm{eV}$ and $532.6 \mathrm{eV}$ corresponding to the $\mathrm{C}$ and $\mathrm{O}$ for SWNT, graphene, A/SWNT and A/graphene (Fig. 5A). A new peak for $\mathrm{N}$ was observed on the A/SWNT and $\mathrm{A} /$ graphene around $399 \mathrm{eV}$, directly demonstrate that adenine is successfully functionalized onto the SWNT and graphene surface. The $\mathrm{N}$ 1s peaks of adenine positively shift by 3.9 and $4.5 \mathrm{eV}$ from $394.6 \mathrm{eV}$ for pure adenine to $398.5 \mathrm{eV}$ and $399.1 \mathrm{eV}$ for $\mathrm{A} / \mathrm{SWNT}$ and $\mathrm{A} / \mathrm{graphene}$ respectively, the significantly positively shift of $\mathrm{N} 1 \mathrm{~s}$ indicates the occurrence of charge transfer from $\mathrm{N}$ species of adenine to SWNT or graphene. The $\mathrm{N}$ amount were calculated from the XPS results and indicate that there are $\sim 2.83$ and 2.1 atom\% for $\mathrm{A} / \mathrm{SWNT}$ and $\mathrm{A} /$ graphene respectively. Further peak deconvolution shows three typical peaks at $398.8,399.3$, and $400.3 \mathrm{eV}$ for A/SWNT, which are correspondent to pyride $(17.4 \%),{ }^{27}$ amine $(62.3 \%)^{27}$ and pyrrolic groups $(20.3 \%)$ in graphitic $\pi$-system. ${ }^{28}$ In the case of A/graphene, there are $398.3,400.1$ and $401.8 \mathrm{eV}$, which is corresponded to pyride (59.6\%), pyrrolic $(28.6 \%)$ and graphitic-N (11.8\%) groups in graphitic $\pi$-system. The results here demonstrate that SWNT and graphene are successfully functionalized by adenine. These $\mathrm{N}$ species exhibit similar binding energy with that of $\mathrm{N}$ species on $\mathrm{N}$ doped carbon materials, ${ }^{27,28}$ indicating formation of ORR active $\mathrm{N}$ species which play a critical role for ORR.

The cyclic voltammograms (CVs) of oxygen reduction in $\mathrm{O}_{2}$ - or $\mathrm{N}_{2}$-saturated 0.1 M KOH solutions on bare SWNT, graphene, A/ SWNT and A/graphene electrodes with a mass loading of $0.25 \mathrm{mg} \mathrm{cm}^{-2}$ are shown in Fig. 6. For bare SWNT and graphene electrode, the onset potential for oxygen reduction is $-0.2375 \mathrm{~V}$, and $-0.21 \mathrm{~V}$ vs. SCE with a single cathodic reduction peak around -0.5 , and $-0.41 \mathrm{~V}$ respectively. After functionalized with adenine, both the onset potential and the potential of cathodic reduction peak of $\mathrm{A} / \mathrm{SWNT}$ have been significantly positively shifted to around $-0.17 \mathrm{~V}$ and $-0.275 \mathrm{~V}$ respectively, indicating the enhancement of ORR activity. In the case of A/graphene, the onset potential and the peak current density remain unchanged, but the cathodic reduction peak shift from $-0.41 \mathrm{~V}$ for graphene to $-0.32 \mathrm{~V}$ for $\mathrm{A} / \mathrm{graphene}$. The obvious reduction peak is likely due to the high surface area of graphene which could absorb oxygen and facilitate the reduction. ${ }^{29}$ These results demonstrate that adenine functionalization can up shift the onset potential and cathodic reduction peak to a certain potential. For comparison, the ORR activity of adenine was tested and the results were shown in Fig. 6C. The results reveal that pure adenine shows similar ORR activity with that of GC electrode, indicating pure adenine is not active for ORR and the enhanced ORR activity of adenine functionalized carbon materials is attributed to the synergistic effect between adenine and SWNT or graphene. 

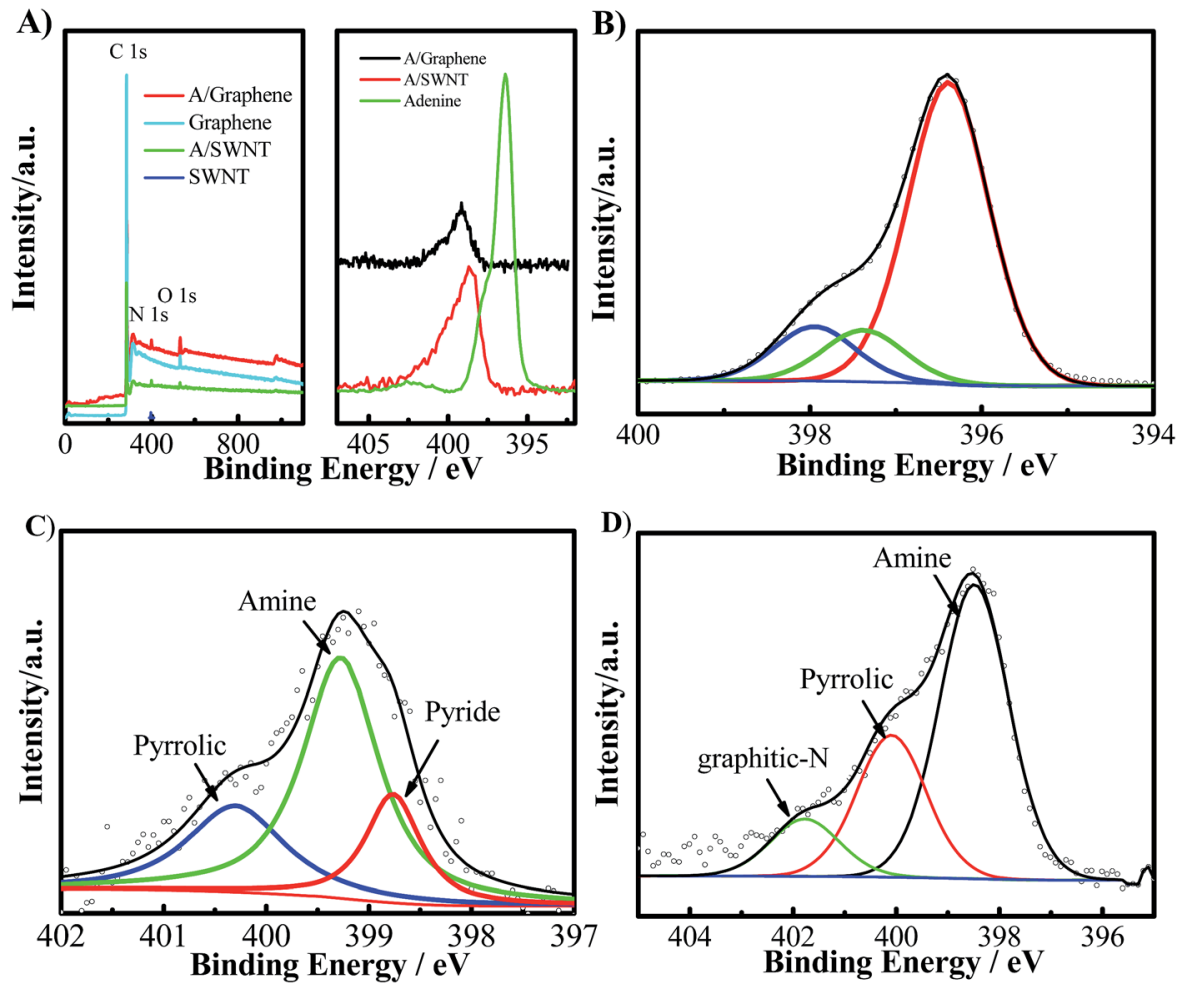

Fig. 5 (A) XPS survey and N 1s spectra; deconvolution of high-resolution spectra of $N$ 1s of (B) adenine, (C) A/SWNT, and (D) A/graphene.
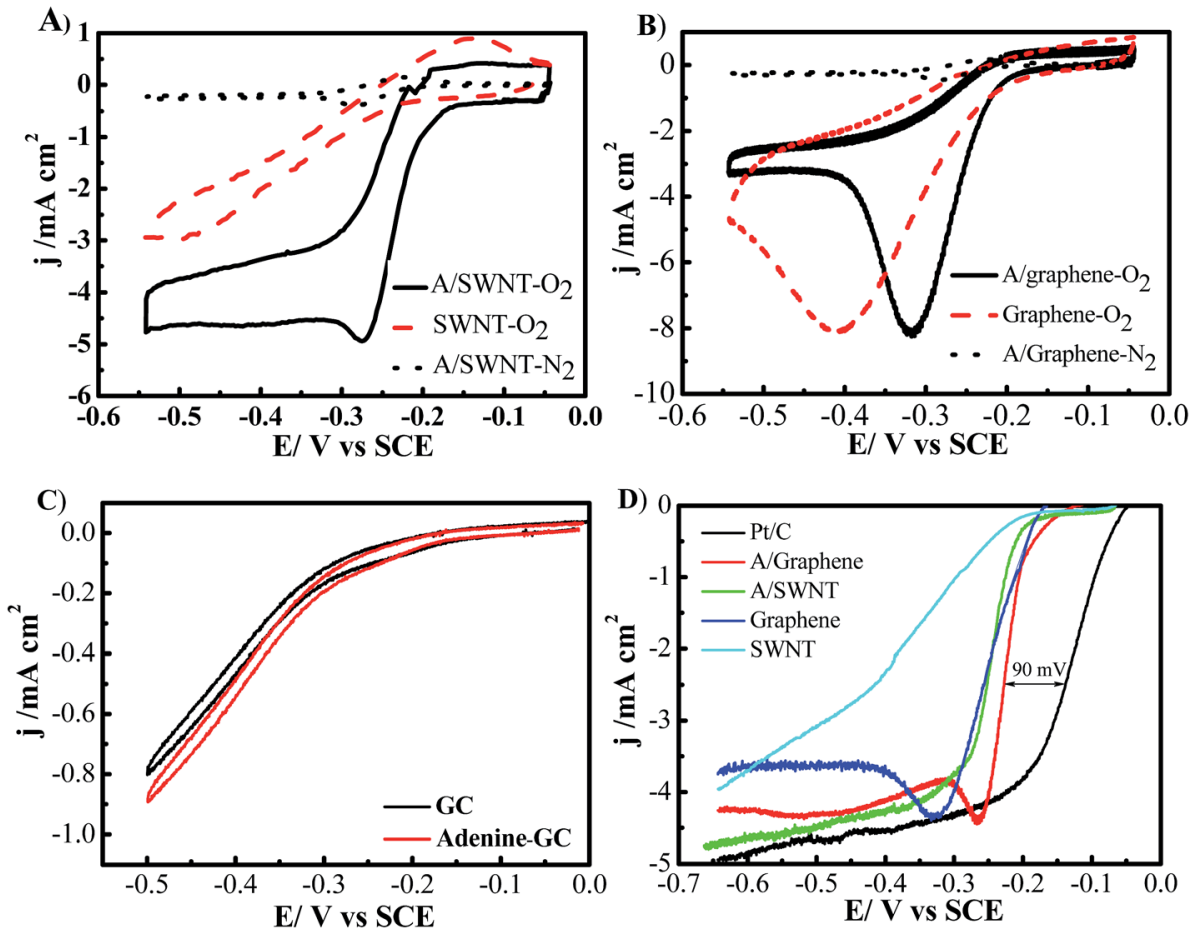

Fig. 6 Cyclic voltammetry curves of ORR on (A) SWNT and A/SWNT in $\mathrm{N}_{2}$ and $\mathrm{O}_{2}$-saturated $0.1 \mathrm{M} \mathrm{KOH}$ solutions, (B) graphene and A/graphene in $\mathrm{N}_{2}$ and $\mathrm{O}_{2}$-saturated $0.1 \mathrm{M} \mathrm{KOH}$ solutions, (C) GC and adenine in $\mathrm{O}_{2}$-saturated $0.1 \mathrm{M} \mathrm{KOH}$ solutions, $\mathrm{CV}$ is conducted at a scan rate of $50 \mathrm{mV} \mathrm{s}^{-1}$. (D) Linear sweep voltammetry curves of ORR in $\mathrm{O}_{2}$-saturated $0.1 \mathrm{M} \mathrm{KOH}$ solution at a scan rate of $10 \mathrm{mV} \mathrm{s}^{-1}$ and rotation rate of 1600 rpm. Carbon based materials loading is $0.25 \mathrm{mg} \mathrm{cm}^{-2}$ and $\mathrm{Pt}-\mathrm{C}$ loading is $0.05 \mathrm{mg} \mathrm{cm}^{-2}$. 
To further investigate the ORR performance, we carried out the linear sweep voltammetry (LSV) measurements on a rotating disk electrode (RDE) for each of the electrode materials with loading of $0.25 \mathrm{mg} \mathrm{cm}{ }^{-2}$ SWNT or graphene-based catalysts or $0.05 \mathrm{mg} \mathrm{cm}^{-2}$ commercial 50\% Pt/C (Alfa Aesar) in $\mathrm{O}_{2}$-saturated $0.1 \mathrm{M} \mathrm{KOH}$ at a scan rate of $10 \mathrm{mV} \mathrm{s}^{-1}$ with a rotation rate of $1600 \mathrm{rpm}$. The ORR is commenced around -0.2375 , and $-0.20 \mathrm{~V}$ for SWNT and graphene respectively (Fig. 6D). And the current density of SWNT keep on increasing linearly, while the current density of graphene electrode continuously increases and then reaches a plateau around $-0.33 \mathrm{~V}$. Adenine functionalization can up shift the onset potential of SWNT by $20 \mathrm{mV}$, and the limiting diffusion current density significantly increased from 0.52 to 2.37 $\mathrm{mA} \mathrm{cm}^{-2}$ at $0.25 \mathrm{~V}$. Remarkably, the half-wave potential positively shifted by $130 \mathrm{mV}$ from $-0.38 \mathrm{~V}$ for SWNT to $-0.25 \mathrm{~V}$ for $\mathrm{A} /$ SWNT. In the case of graphene and A/graphene electrode, the limiting diffusion current density reaches a peak around $-0.33 \mathrm{~V}$ and $-0.26 \mathrm{~V}$ respectively with a similar limiting current density of $4.5 \mathrm{~mA} \mathrm{~cm} \mathrm{~cm}^{-2}$. And the ORR half-wave potential is positively shifted by $30 \mathrm{mV}$ from $-0.25 \mathrm{~V}$ for graphene to $-0.22 \mathrm{~V}$ for $\mathrm{A} /$ graphene. Although the onset potential of adenine functionalized SWNT is still about $90 \mathrm{mV}$ more negative than of commercial $50 \% \mathrm{Pt} / \mathrm{C}$, the results reveal that adenine functionalization can up shift the onset potential to certain value and significantly increase the limiting current density of SWNT and graphene. Adenine functionalized graphene show better ORR activity compared with A/SWNT, which is because of the strong interaction between adenine and graphene, leads to formation of larger amount of ORR active $\mathrm{N}$ species, such as pyrrolic and graphitic $\mathrm{N}$ species (Fig. 5D). The performance of A/SWNT and A/graphene show higher performance compared with reported polymer functionalized carbon materials for ORR. ${ }^{\mathbf{1 8 , 2 3 , 2 9}}$ For example, the
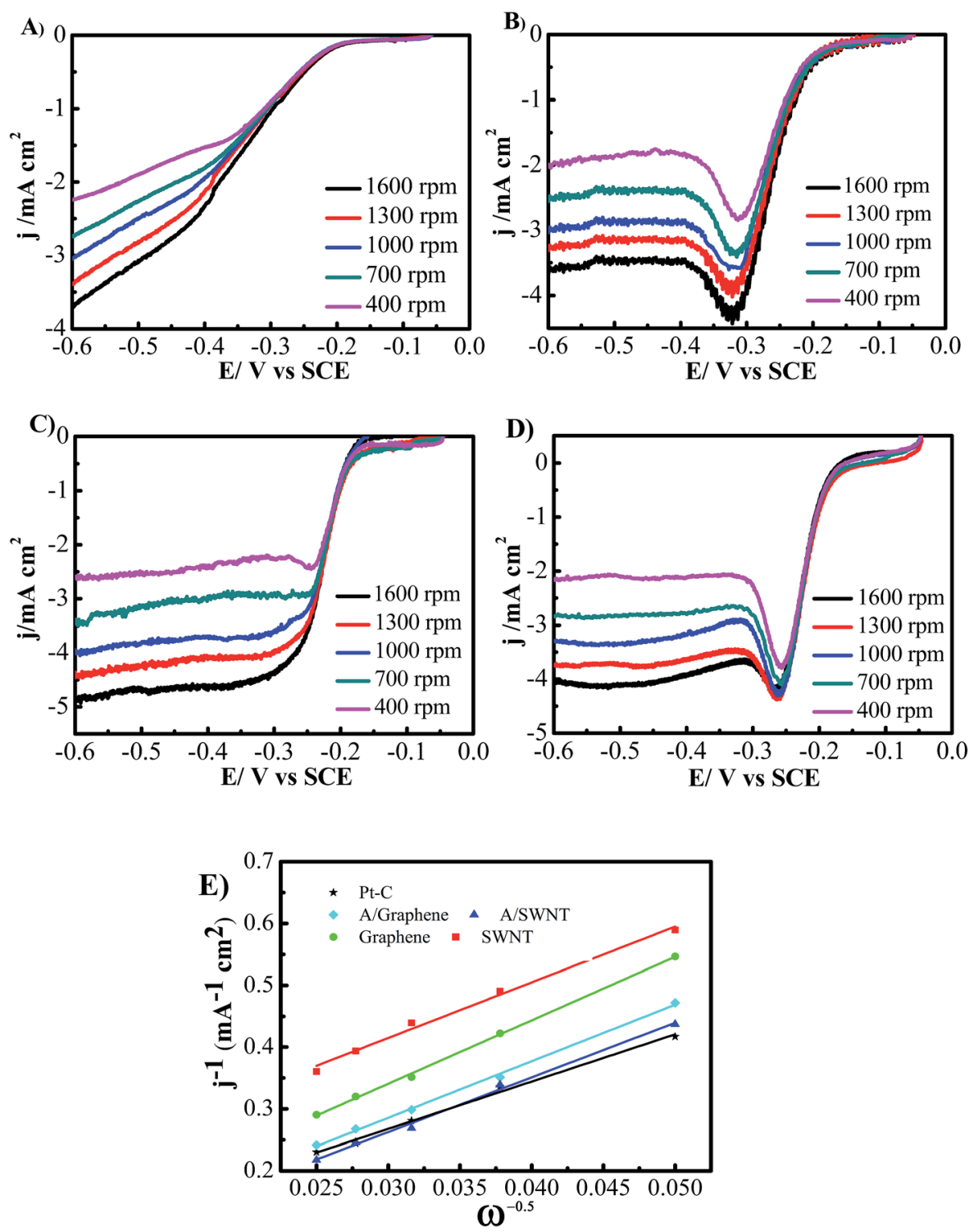

Fig. 7 Linear sweep voltammetry curves of ORR at various rotation rates on (A) SWNT, (B) graphene, (C) A/SWNT, and (D) A/graphene in $\mathrm{O}_{2}$ saturated $0.1 \mathrm{M} \mathrm{KOH}$ solution with a scan rate of $10 \mathrm{mV} \mathrm{s}^{-1}$. (E) The Koutecky-Levich plots at various rotation rates on SWNT, graphene, A/SWNT, and $\mathrm{A}$ /graphene and $\mathrm{Pt}-\mathrm{C}$ electrode at $-0.45 \mathrm{~V}$ in $\mathrm{O}_{2}$-saturated $0.1 \mathrm{M} \mathrm{KOH}$ solution. Carbon based materials loading is $0.25 \mathrm{mg}^{-2}$. 
onset potential and half-wave potential is $-0.18 \mathrm{~V}$ and $-0.33 \mathrm{~V} v s$. SCE for PDDA functionalized carbon nanotubes, ${ }^{23}$ which is higher than that of $-0.17 \mathrm{~V}$ and $-0.25 \mathrm{~V} v s$. SCE in the case of A/ SWNT respectively. These results reveal adenine functionalization could be a promise approach to improve the ORR activity of carbon materials.

The LSV measurements at different rotating rate were also carried out to gain further insight on the ORR performance of the SWNT and graphene electrodes before and after functionalized with adenine (Fig. 7). The limiting current densities of all the electrodes are enhanced with the increase of rotating rate. The results show that the limiting current densities of adenine functionalized SWNT and graphene are much higher than that without functionalization at corresponding rotating rate, which is consistent with the significant better activity for ORR obtain from CVs and LSVs. The transferred electron number per oxygen molecule involved in the oxygen reduction at electrode was determined by the Koutecky-Levich equation given below: ${ }^{23}$

$$
\frac{1}{j}=\frac{1}{j_{\mathrm{k}}}+\frac{1}{B \omega^{0.5}}
$$

where $j_{\mathrm{k}}$ is the kinetic current and $\omega$ is the electrode rotating rate. $B$ could be determined from the slope of $\mathrm{K}-\mathrm{L}$ plots based on Levich equation as follows:

$$
B=0.2 n F\left(D_{\mathrm{O}_{2}}\right)^{2 / 3} \nu^{-1 / 6} C_{\mathrm{O}_{2}}
$$

where $n$ represents the number of electrons transferred per oxygen molecule, $F$ is the Faraday constant $(F=96485 \mathrm{C}$ $\left.\mathrm{mol}^{-1}\right), D_{\mathrm{O}_{2}}$ is the diffusion coefficient of $\mathrm{O}_{2}$ in $0.1 \mathrm{M} \mathrm{KOH} \mathrm{(1.9}$ $\left.\times 10^{-5} \mathrm{~cm}^{2} \mathrm{~s}^{-1}\right), \nu$ is the kinetic viscosity $\left(0.01 \mathrm{~cm}^{2} \mathrm{~s}^{-1}\right)$, and $C_{\mathrm{O}_{2}}$ is the bulk concentration of $\mathrm{O}_{2}\left(1.2 \times 10^{-6} \mathrm{~mol} \mathrm{~cm}^{-3}\right)$. The constant 0.2 is adopted when the rotation speed is expressed in rpm. The Koutecky-Levich plots indicate that ORR on both SWNT and graphene exhibits a two electron consistent with the reported results ${ }^{30}$ (Fig. 7E). The electron transfer number is $\sim 4$ for $20 \% \mathrm{Pt} / \mathrm{C}$, which is significantly higher than that of SWNT and graphene $(n=2)$. The electron transfer number on A/SWNT and $\mathrm{A} /$ graphene electrode was calculated $\sim 3$, which indicated that the ORR on A/SWNT and A/graphene electrode is a combination of two electrons and four electrons pathway.

Fuel crossover and CO poisoning of Pt base catalysts are the major challenges of polymer electrolyte fuel cells. For example, methanol crossover during DMFC operation results in low power output because chemical oxidation of methanol at the cathode leads to electrode depolarization, mixed potential (resulting in the open-circuit voltage of the DMFC below $0.8 \mathrm{~V}$ ), consumption of $\mathrm{O}_{2}$, cathode catalyst poisoning by $\mathrm{CO}$ (an intermediate of methanol oxidation), and serious water accumulation on the cathode (limits $\mathrm{O}_{2}$ access to cathode catalyst sites). ${ }^{31}$ Here, the possible cross over of methanol fuels and poisoning of carbon monoxide (CO) were also tested by addition of methanol and bubbling with $\mathrm{CO}$ separately during the process of chronoamperometry at $-0.4 \mathrm{~V}$ in $\mathrm{O}_{2}$ saturated $0.1 \mathrm{~mol} \mathrm{~L}^{-1} \mathrm{KOH}$ solution. As it is shown in Fig. 8, a sharp decrease in current density was observed on $\mathrm{Pt} / \mathrm{C}$ electrode instantly after addition of $1.0 \mathrm{~mL}$ methanol or bubbling with $\mathrm{CO}$

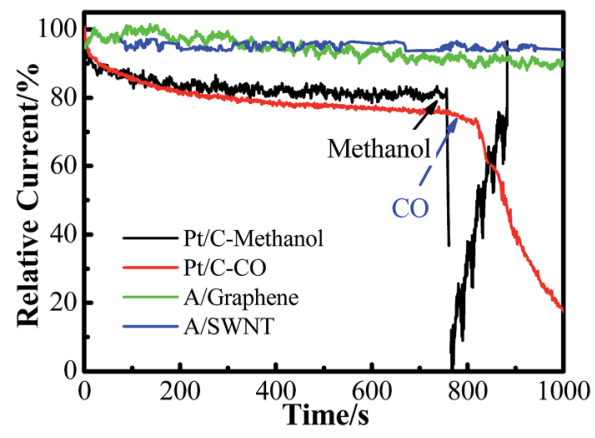

Fig. 8 Chronoamperometry at $-0.4 \mathrm{~V}$ of $\mathrm{Pt} / \mathrm{C}$ and adenine functionalized SWNT and graphene in $0.1 \mathrm{M} \mathrm{KOH}$ solution with addition of 1 $\mathrm{mL}$ methanol and CO. Carbon based materials loading is $0.25 \mathrm{mg}$ $\mathrm{cm}^{-2}$ and Pt-C loading is $0.05 \mathrm{mg} \mathrm{cm}^{-2}$.

Table 1 Summary of the properties, onset potential and half-wave potential and electron transfer number of SWNT, graphene, functionalized SWNT and graphene

\begin{tabular}{lllll}
\hline Materials & SWNT & Graphene & A/SWNT & A/graphene \\
\hline Surface area $\left(\mathrm{g} \mathrm{m}^{-2}\right)$ & 651 & 504 & - & - \\
$I_{\mathrm{G}} / I_{\mathrm{D}}$ & 6.4 & 0.38 & 7.3 & 0.44 \\
Onset potential $(\mathrm{V})$ & -0.2375 & -0.21 & -0.17 & -0.21 \\
Half-wave potential & -0.38 & -0.25 & -0.25 & -0.22 \\
Electron transfer number & 2 & 2 & 3 & 3
\end{tabular}

due to the competition oxidation of methanol with $\mathrm{ORR}^{32,33}$ or the CO poisoning of $\mathrm{Pt}$ active sites in the case of $\mathrm{CO} .{ }^{34}$ However, the corresponding chronoamperometric responses for the functionalized graphene electrode remains unchanged after the addition of methanol and CO, proved that adenine functionalized SWNT has better selectivity toward $\mathrm{O}_{2}$ reduction.

Adenine is inactive for ORR, however adenine functionalized SWNT and graphene show significantly enhanced activity for ORR with a combination of two electron and 4 electron process due to the interaction of adenine and carbon plane (Table 1). And the A/SWNT and A/graphene exhibits better fuel selectivity, better $\mathrm{CO}$ tolerance and longer stability than commercially available Pt/ $\mathrm{C}$ electrode. The ORR activity of adenine functionalized carbon material can attribute to the $\pi-\pi$ interaction of the nitrogen groups of adenine with SWNT or graphene, and the XPS results indicate the charge transfer from nitrogen atoms of adenine to the conjugated graphitic $\pi$-system, which appears to impart a relatively high positive charge density to form pyrrolic and graphitic like $\mathrm{N}$ groups. At the same time, the charge transfer also perturbs the homogeneous $\pi$-electron cloud to change the electronic surface state of the CNT and graphene. These electrons accepting $\mathrm{N}$ incorporated with SWNT plane and graphene provide active sites for ORR, which enable the adenine functionalized SWNT and graphene with enhanced ORR activity.

\section{Conclusions}

Adenine functionalized SWNT and graphene can enhance the ORR of SWNT and graphene with better fuel selectivity, and 
better CO tolerance and longer stability. The ORR activity of adenine functionalized SWNT and graphene can attribute to the $\pi-\pi$ interaction of adenine with SWNT or graphene. The SWNT or graphene can draw the electrons from the nitrogen species in adenine to form positively charged pyrrolic/graphitic-N-type nitrogen groups as ORR active sites, similar with that of $\mathrm{N}$ doped carbon materials. The results demonstrate that molecular functionalization of carbon materials can actually provide active sites for ORR similar with that of N-doping carbon materials, which offers a simple and straight forward method to improve the ORR activity of SWNT and graphene.

\section{Acknowledgements}

This work was supported by Collaborative Innovation Center of New Chemical Technologies for Environmental Benignity and Efficient Resource Utilization and Opening Foundation of the Chinese National Engineering Research Center for Control and Treatment of Heavy metal Pollution, Changsha, 410083, China (No. 2015CNERC-CTHMP-).

\section{References}

1 R. Bashyam and P. Zelenay, Nature, 2006, 443, 63.

2 M. K. Debe, Nature, 2012, 486, 43-51.

3 M. Lefèvre, E. Proietti, F. Jaouen and J. P. Dodelet, Science, 2009, 324, 71-74.

4 K. A. Kuttiyiel, K. Sasaki, Y. Choi, D. Su, P. Liu and R. R. Adzic, Nano Lett., 2012, 12, 6266.

5 H. Y. Lee, W. Vogel and P. J. Chu, Langmuir, 2013, 27, 14654. 6 V. R. Stamenkovic, B. Fowler, B. S. Mun, G. Wang, P. N. Ross, C. A. Lucas and N. M. Marković, Science, 2007, 315, 493-497.

7 D. Wang, H. L. Xin, R. Hovden, H. Wang, Y. Yu, D. A. Muller, F. J. Disalvo and H. D. Abruña, Nat. Mater., 2012, 12, 81-87. 8 H. Chen, Y. Yu, H. L. Xin, K. A. Newton, M. E. Holtz, D. Wang, D. A. Muller, H. D. Abruña and F. J. Disalvo, Chem. Mater., 2013, 25, 1436-1442.

9 S. Koh and P. Strasser, J. Am. Chem. Soc., 2007, 129, 12624.

10 P. Mani, A. Ratndeep Srivastava and P. Strasser, J. Phys. Chem. C, 2009, 112, 2770-2778.

11 P. Strasser, S. Koh, T. Anniyev, J. Greeley, K. More, C. Yu, Z. Liu, S. Kaya, D. Nordlund and H. Ogasawara, Nat. Chem., 2010, 2, 454.

12 D. Wang, P. Zhao and Y. Li, Sci. Rep., 2011, 1, 37.
13 L. Dai, Y. Xue, L. Qu, H. J. Choi and J. B. Baek, Chem. Rev., 2015, 115, 4823.

14 Z. Chen, D. Higgins, H. Tao, R. S. Hsu and Z. Chen, J. Phys. Chem. C, 2009, 113, 21008-21013.

15 K. Gong, F. Du, Z. Xia, M. Durstock and L. Dai, Science, 2009, 323, 760-764.

16 L. Yang, S. Jiang, Y. Zhao, L. Zhu, S. Chen, X. Wang, Q. Wu, J. Ma, Y. Ma and Z. Hu, Angew. Chem., Int. Ed., 2011, 123, $7132-7135$.

17 Z. Xu, H. Li, M. Fu, H. Luo, H. Sun, L. Zhang, K. Li, B. Wei, J. Lu and X. Zhao, J. Mater. Chem., 2012, 22, 18230-18236.

18 R. Kannan, U. Bipinlal, S. Kurungot and V. K. Pillai, Phys. Chem. Chem. Phys., 2011, 13, 10312-10317.

19 Y. J. Yang, Fullerenes, Nanotubes, Carbon Nanostruct., 2016, 24, 144-148.

20 S. Wang, ACS Nano, 2011, 5, 6202-6209.

21 S. Wang, D. Yu and L. Dai, J. Am. Chem. Soc., 2011, 133, 5182. 22 L. Dai, Y. Xue, L. Qu, H.-J. Choi and J.-B. Baek, Chem. Rev., 2015, 115, 4823-4892.

23 S. Wang, D. Yu and L. Dai, J. Am. Chem. Soc., 2011, 133, 51825185.

24 Y. Cheng, C. Xu, L. Jia, J. D. Gale, L. Zhang, C. Liu, P. K. Shen and S. P. Jiang, Appl. Catal., B, 2015, 163, 96-104.

25 U. J. Kim, C. A. Furtado, X. Liu, G. Chen and P. C. Eklund, J. Am. Chem. Soc., 2005, 127, 15437.

26 S. Saito and A. Zettl, Carbon Nanotubes: Quantum Cylinders of Graphene, Elsevier Science Bv, Amsterdam, 2008.

27 K. Artyushkova, B. Kiefer, B. Halevi, A. Knop-Gericke, R. Schlogl and P. Atanassov, Chem. Commun., 2013, 49, 2539.

28 Y. Zheng, Y. Jiao, L. Ge, M. Jaroniec and S. Z. Qiao, Angew. Chem., 2013, 52, 3110.

29 S. Wang, D. Yu, L. Dai, D. W. Chang and J.-B. Baek, ACS Nano, 2011, 5, 6202-6209.

30 C. Song and J. Zhang, PEM Fuel Cell Electrocatalysts and Catalyst Layers, Springer, London, 2008.

31 M. Ahmed and I. Dincer, Int. J. Energy Res., 2011, 35, 12131228.

32 A. Suleiman, C. L. Menendez, R. Polanco, E. R. Fachini, Y. Hernandez-Lebron, M. J. F. Guinel, R. Roque-Malherbe and C. R. Cabrera, RSC Adv., 2015, 5, 7637-7646.

33 B. Singh, L. Murad, F. Laffir, C. Dickinson and E. Dempsey, Nanoscale, 2011, 3, 3334-3349.

34 J. J. Baschuk and X. Li, Int. J. Energy Res., 2001, 25, 695-713. 